\title{
Intravenous lidocaine infusions for 48 hours in open colorectal surgery: a prospective, randomized, double-blinded, placebo-controlled trial
}

\author{
Matthew Liang Jinn $\mathrm{Ho}^{1}$, Stephen John Kerr ${ }^{2}$, and Jennifer Stevens ${ }^{3}$ \\ ${ }^{1}$ Department of Anaesthesia, Concord Repatriation General Hospital, Camperdown NSW, ${ }^{2}$ The Kirby Institute, \\ University of New South Wales, Kensington NSW, ${ }^{3}$ Department of Anaesthesia, St Vincent's Hospital, Darlinghurst \\ NSW, Sydney, Australia
}

Background: Although intravenous (i.v.) lidocaine is used as a perioperative analgesic in abdominal surgery, evidence of efficacy is limited. The infusion dose and duration remain unclear. This study aimed to investigate the effect of a longer low-dose 48-hour infusion regimen on these outcomes.

Methods: Fifty-eight adults undergoing elective open colorectal surgery were randomized into the lidocaine group (1.5 $\mathrm{mg} / \mathrm{kg}$ bolus followed by $1 \mathrm{mg} / \mathrm{kg} / \mathrm{h}$ infusion for 48 hours) and control group. After surgery, patients were given a fentanyl patient-controlled analgesia machine and time to first bowel movement (primary outcome) and flatus were recorded. Postoperative pain scores and fentanyl consumption were assessed for 72 hours.

Results: There was no significant difference in time to first bowel movement $(80.1 \pm 42.2$ vs. $82.5 \pm 40.4$ hours; $P=0.830)$, time to first flatus ( $64.7 \pm 38.5$ vs. $70.0 \pm 31.2$ hours; $\mathrm{P}=0.568)$, length of hospital stay (9 [8-13] vs. 11 [9-14) days; $\mathrm{P}=$ $0.531]$, nor postoperative pain scores in the lidocaine vs. control groups. Cumulative opioid consumption was significantly lower in the lidocaine vs. the control group from 24 hours onwards. At 72 hours, cumulative opioid consumption ( $\mu \mathrm{g}$ fentanyl) in the lidocaine group $(1,570$ [825-3,587]) was over $40 \%$ lower than in the placebo group $(2,730$ [1,778-5,327]; $\mathrm{P}=0.039$ ).

Conclusions: A 48-hour low-dose i.v. lidocaine infusion does not significantly speed the return of bowel function in patients undergoing elective open colorectal surgery. It was associated with reduced postoperative opioid consumption, but not with earlier hospital discharge, or lower pain scores.

Keywords: Colorectal surgery; Ileus; Lidocaine; Local anesthetics; Opioid analgesics; Postoperative pain.

Corresponding author: Jennifer Stevens, M.D.

Department of Anaesthesia, St Vincent's Hospital, Level 4, 390, Victoria Street, Darlinghurst NSW 2010, Australia

Tel: 61-2-8382-3200, Fax: 61-2-8382-3981

Email: jen_a_stevens@hotmail.com

ORCID: https://orcid.org/0000-0001-9837-0031

Received: August 31, 2017.

Revised: October 28, 2017 (1st); November 19, 2017 (2nd).

Accepted: November 21, 2017.

Korean J Anesthesiol 2018 February 71(1): 57-65

https://doi.org/10.4097/kjae.2018.71.1.57

\section{Introduction}

Open abdominal surgery is associated with post-operative pain, nausea, ileus, and prolonged hospital stay with associated costs $[1,2]$. While opioids have been the mainstay of peri-operative analgesia, they are significantly associated with post-operative ileus, especially when daily dosing exceeds $2 \mathrm{mg}$ of intravenous (i.v.) hydromorphone equivalents [3]. This has led to the use of alternative modes of analgesia [1], including epi-

(c) This is an open-access article distributed under the terms of the Creative Commons Attribution Non-Commercial License (http://creativecommons.org/ licenses/by-nc/4.0/), which permits unrestricted non-commercial use, distribution, and reproduction in any medium, provided the original work is properly cited. 
dural anesthesia and transversus abdominis plane catheters [2]. Intravenous lidocaine is a less invasive and costly alternative for patients not willing, or unable to undergo these procedures.

Sun et al. [4] recently undertook a meta-analysis of i.v. lidocaine in fifteen placebo-controlled trials in open abdominal surgery. In most studies, the scope of assessment included bowel function, length of stay, pain scores, or opioid consumption. Some, but not all studies report benefits in some of these outcome measures. In the 3 of 15 studies that assessed all these measures, the benefits of lidocaine compared to conventional opioid analgesia were equivocal [5-7]. The regimens were variable, with most employing a loading dose of 1-2 mg/ $\mathrm{kg}$ followed by an infusion of $1-3 \mathrm{mg} / \mathrm{kg} / \mathrm{h}$ usually at the start of surgery, continuing for up to 24 hours postoperatively [4].

In this study, we tested the hypothesis that a longer infusion regimen of 48 hours would be associated with a greater effect on bowel function in patients undergoing open abdominal surgery. We also aimed to evaluate the effect of this regimen on hospital length of stay, pain scores, and opioid use.

\section{Materials and Methods}

\section{Study protocol}

This was a two-center prospective, randomized (1:1), double-blinded, placebo-controlled parallel group trial conducted in Sydney, Australia between 4th March 2014 and 22nd July 2015.

\section{Ethics approval/license}

The study was approved by the St Vincent's Hospital Human Resources and Ethics Committee (ref: HREC/12/SVH/297) and registered at http://www.ANZCTR.org.au under the Australian New Zealand Clinical Trials Registry (ref: ACTRN12616000478415).

\section{Patient population}

Fifty-eight adult patients aged 18-80 undergoing elective open colorectal surgery were enrolled in the study. The study was conducted at two affiliated tertiary health care centers: St Vincent's Public Hospital and St Vincent's Private Hospital, Sydney. Patients were under the care of three colorectal surgeons who operated at both centers. Exclusion criteria were: American Society of Anesthesiologists classification status IV or above; liver cirrhosis; severe chronic renal impairment (serum creatinine $>200$ umol/L); pregnancy; cardiac conduction abnormalities; congestive heart failure; epilepsy; allergy to any of the study medications; opioid or alcohol abuse; and reported bowel movement frequencies greater than three per day, or less than three per week.

\section{Conduct of study}

Patients were allocated to two groups (lidocaine or control) by the statistician using a computer-generated randomization number list in Microsoft Excel with a $1: 1$ ratio, in blocks of four. This allocation sequence was concealed from the study team and kept in an opaque envelope in the locked possession of the in-charge operating room nurse (not otherwise involved in the study). Following informed written consent at the pre-anesthetic clinic, patients received a study enrolment number. On the morning of surgery, the in-charge nurse checked the allocation sequence and prepared the enrolled patient's corresponding infusion (lidocaine or saline). All investigators, treating staff, participants, outcome adjudicators, and data collectors were blinded to the intervention.

Standard peri-operative monitoring included a continuous electrocardiogram, non-invasive arterial pressure measurement, and pulse oximetry. Anesthesia was induced to facilitate orotracheal intubation, according to the discretion of the blinded attending anesthetist. The lidocaine group received $1.5 \mathrm{mg} / \mathrm{kg}$ over $5 \mathrm{~min}$ (lidocaine $2.5 \%, 0.06 \mathrm{ml} / \mathrm{kg}$ ) immediately post-induction, followed by $1 \mathrm{mg} / \mathrm{kg} / \mathrm{h}$ (lidocaine $2.5 \%, 0.04 \mathrm{ml} / \mathrm{kg} /$ h) and the control group received an equal volume and rate of normal saline through a dedicated i.v. line. This infusion was continued for 48 hours post-bolus dose. In accordance with current safety standards in Australia for i.v. lidocaine infusions, all patients received continuous three-lead ECG monitoring for the duration of the study drug infusion (48 hours).

All patients at St Vincent's Public Hospital (29 of 58) had plasma lidocaine levels measured one hour after bolus to validate the accuracy of study blinding. Anesthesia was maintained with sevoflurane or desflurane (no nitrous oxide), with positive pressure ventilation in a circle system. I.V. fentanyl was used as the sole opioid analgesic in all patients, and other multimodal agents (non-steroidal anti-inflammatory drugs, ketamine) were given at the discretion of the anesthetist. Patients did not receive any additional local anesthetic peri-operatively. All patients received nasogastric tubes intra-operatively, which remained in-situ until first bowel movement.

On arrival at the post-anesthesia care unit (PACU), all patients were connected to an i.v. fentanyl patient-controlled analgesia (PCA) pump (20 $\mu$ g bolus, 5 min lockout) for 72 hours. PCA fentanyl consumption and numerical pain rating scale (NRS, 0-10) at rest were recorded by a blinded non-study nurse at $30 \mathrm{~min}, 1,2,4,8,12,24,36,48,60$, and 72 hours post-surgery. A blinded non-study pain physician adjusted the PCA by increasing the bolus dose according to their discretion if the pain was not controlled (NRS score $>6$ ). The nurse caring for the patient filled out a questionnaire documenting the time of return of first bowel movement and flatus, and adverse effects. 


\section{Data analysis}

To detect a difference in mean time to first bowel movement of 12 hours, a sample size of 28 patients in each group was calculated as sufficient, with $80 \%$ power at a two-tailed $5 \%$ significance level. This estimation was based on a meta-analysis [4] reporting a reduction in mean time to first bowel movement by 15.1 hours. Twenty-nine patients per group were enrolled to compensate for possible dropouts.

Statistical analysis was conducted with Stata 14 (Statacorp, College Station, TX, USA). The primary outcome measure was time to first bowel movement. Secondary outcome measures were time to first flatus, duration of hospital stay, pain scores, and cumulative opioid use up to 72 hours postoperatively. The normality of study endpoint distributions was assessed graphically and with the Shapiro-Wilk test. Normally distributed variables were reported as mean (SD) and comparisons made by calculating the mean difference and 95\% CI between the lidocaine and placebo arms. Non-normally distributed data were reported as the median and inter-quartile range (IQR) and analyzed using the Wilcoxon test. Postoperative pain scores and cumulative opioid use were compared between the treatment arms at 1, 4, $12,24,48$, and 72 hours postoperatively. Categorical characteristics were summed as $\mathrm{n}(\%)$ and analyzed using a chi-square or Fisher's exact test, as appropriate. A formal comparison of the postoperative pain scores over the total duration of follow-up was made with a generalized estimating equation (GEE).

\section{Results}

\section{Baseline characteristics}

Seventy-three patients were screened, of whom 18 were not eligible (Fig. 1). Fifty-eight patients, 42 of whom were male, were randomized (29 each to lidocaine and control arms). The median patient age was $64(51-70)$ years, and body weight was 75 (64-89) kg. Patient characteristics were similar between the two groups (Table 1). The presence of inflammatory bowel disease, stoma formation, surgical duration, and intra-operative opioid consumption were similar between the two groups. One patient in the lidocaine group had the infusion discontinued by the anesthetist, due to transient ST segment depression at induction of anesthesia and was excluded from analysis.

\section{Bowel recovery}

Time to recovery of bowel function, first flatus, and duration of hospital stay are shown in Table 2. The mean time to first bowel movement of 80.1 (42.2) hours in the lidocaine arm did not differ significantly from 82.5 (40.4) hours in the placebo arm. There was also no significant difference in mean time to first flatus between the lidocaine arm $(64.7 \pm 38.5$ hours $)$ and the placebo arm $(70.0 \pm 31.2$ hours; $\mathrm{P}=0.568)$. The median length of stay of 9 (8-13) days in the lidocaine arm did not differ significantly from $11(9-14)$ days in the placebo arm $(\mathrm{P}=0.531)$.

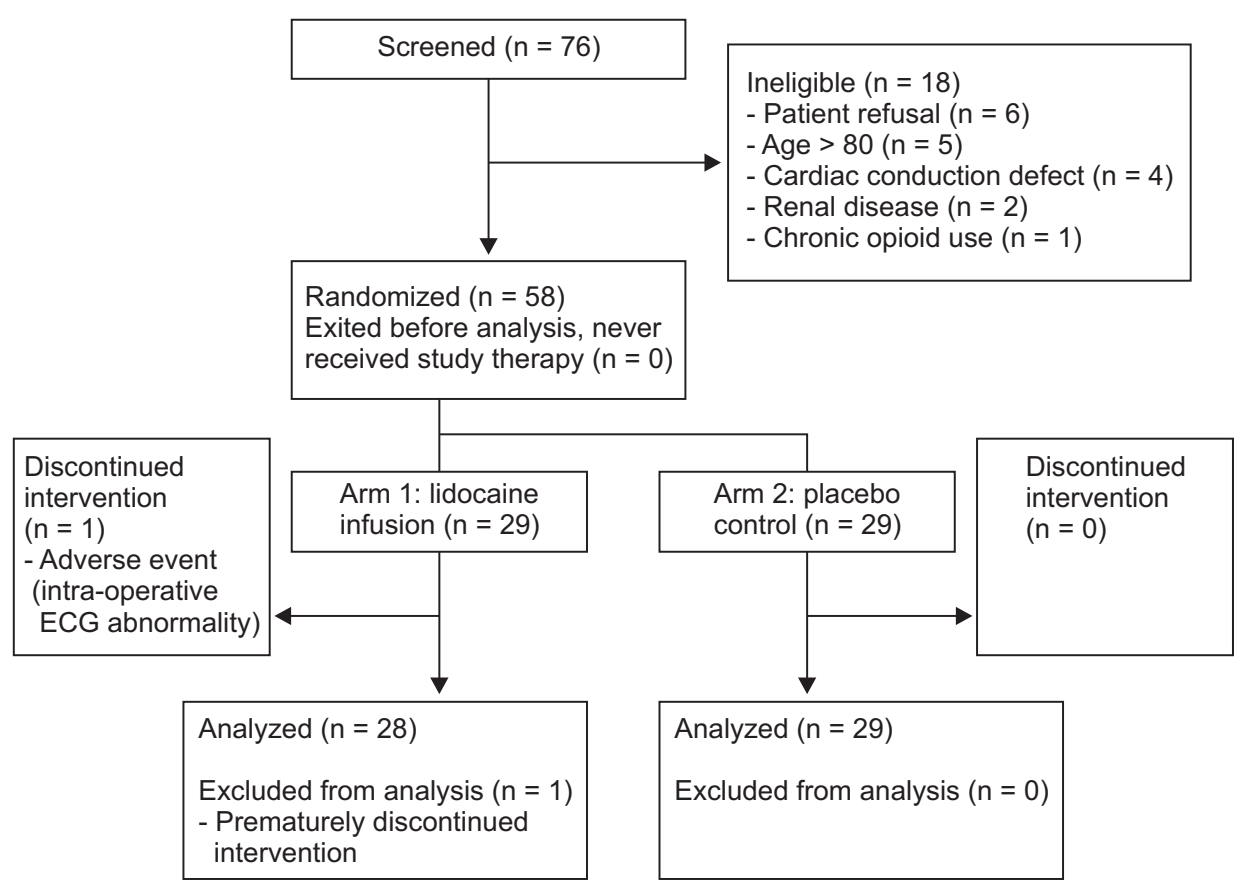

Fig. 1. Study design according to the CONSORT Statement. 


\section{Pain scores and opioid use}

All NRS scores at rest between $30 \mathrm{~min}$ and 72 hours after surgery did not differ significantly between randomized arms (Fig. 2). The mean difference in pain scores over the first 72 hours analyzed by a GEE, did not differ significantly between the lidocaine and the placebo groups $(-0.21,95 \% \mathrm{CI}-1.02$ to 0.59 ; $\mathrm{P}=$ $0.61)$.

The cumulative fentanyl opioid consumption for the first 72 postoperative hours is shown in Fig. 3. The lidocaine group had significantly lower cumulative opioid use compared to the placebo group at all measured points between 24 and 72 hours postoperatively $(\mathrm{P}<0.05)$, with a reduction of over $40 \%$ at 72 hours $[1,570(825-3,587) \mu \mathrm{g}$ vs. $2,730(1,778-5,327) \mu \mathrm{g} ; \mathrm{P}=$ 0.039]. The maximum median daily opioid consumption for

Table 1. Patient Characteristics and Clinical Data

\begin{tabular}{|c|c|c|}
\hline Variables & $\begin{array}{l}\text { Placebo } \\
(\mathrm{n}=29)\end{array}$ & $\begin{array}{l}\text { Lidocaine arm } \\
\qquad(\mathrm{n}=28)\end{array}$ \\
\hline \multicolumn{3}{|l|}{ Patients } \\
\hline Age (yr) & $66(62-70)$ & $57(47-70)$ \\
\hline Female & $8(28)$ & $8(29)$ \\
\hline Weight (kg) & $76(68-92)$ & $74.5(59-82.5)$ \\
\hline Inflammatory bowel disease & $4(14)$ & $4(14)$ \\
\hline \multicolumn{3}{|l|}{ ASA scores } \\
\hline 1 & $0(0)$ & $1(4)$ \\
\hline 2 & $19(66)$ & $22(79)$ \\
\hline 3 & $10(34)$ & $5(18)$ \\
\hline \multicolumn{3}{|l|}{ Operation } \\
\hline Surgery duration (min) & $328(112)$ & $326(96)$ \\
\hline $\begin{array}{l}\text { Intra-operative opioid use } \\
(\mu \mathrm{g} \text { fentanyl) }\end{array}$ & $500(450-600)$ & $500(400-650)$ \\
\hline PACU opioid use ( $\mu$ g fentanyl) & $160(100-280)$ & $190(100-320)$ \\
\hline \multicolumn{3}{|l|}{ Operation type } \\
\hline Stoma formation & $12(41)$ & $16(57)$ \\
\hline Anterior resection & $10(34)$ & $14(50)$ \\
\hline Right hemicolectomy & $10(34)$ & $4(14)$ \\
\hline Left hemicolectomy & $1(3)$ & $1(4)$ \\
\hline Total colectomy & $3(10)$ & $2(7)$ \\
\hline Abdominoperineal resection & $2(7)$ & $2(7)$ \\
\hline Proctocolectomy & $1(3)$ & $3(11)$ \\
\hline Other & $2(7)$ & $2(7)$ \\
\hline
\end{tabular}

Data are presented as mean (SD) for surgery duration, median (IQR) for other continuous variables, or $\mathrm{n}(\%)$ for categorical variables. ASA: American Society of Anesthesiologists physical classification. both groups was well in excess of the $2 \mathrm{mg}$ i.v. hydromorphone equivalents ( $100 \mu \mathrm{g}$ i.v. fentanyl) predisposing to postoperative ileus [3], but was significantly lower in the lidocaine group [993 (513-1,620) $\mu$ g vs. $1,406(1,120-2,488) \mu g$ for placebo, $\mathrm{P}=0.027]$. Cumulative opioid consumption was not significantly different between $15 \mathrm{~min}$ and 12 hours postoperatively.

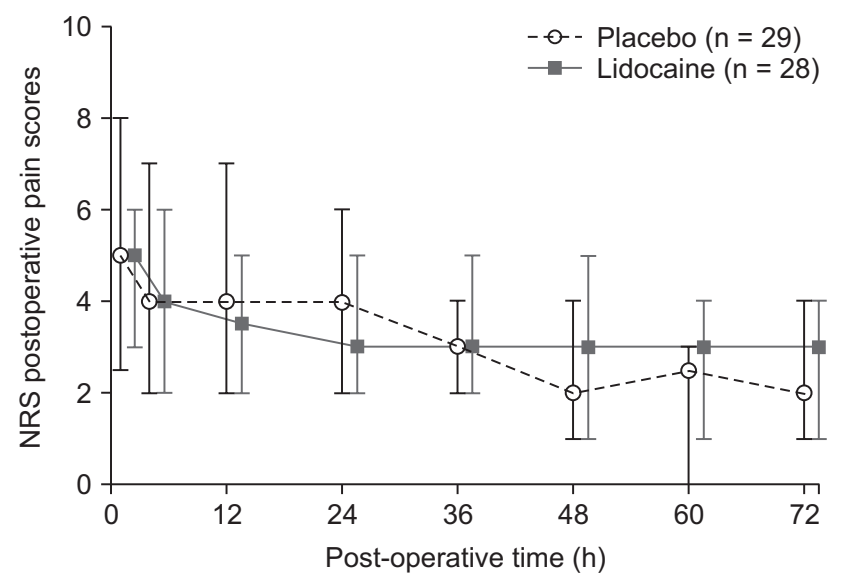

Fig. 2. Postoperative NRS pain scores (0-10). Data are presented as median (IQR). None of the differences in median NRS scores reached clinical significance at any time during the first 72 postoperative hours $(\mathrm{P}$ $>0.05)$.

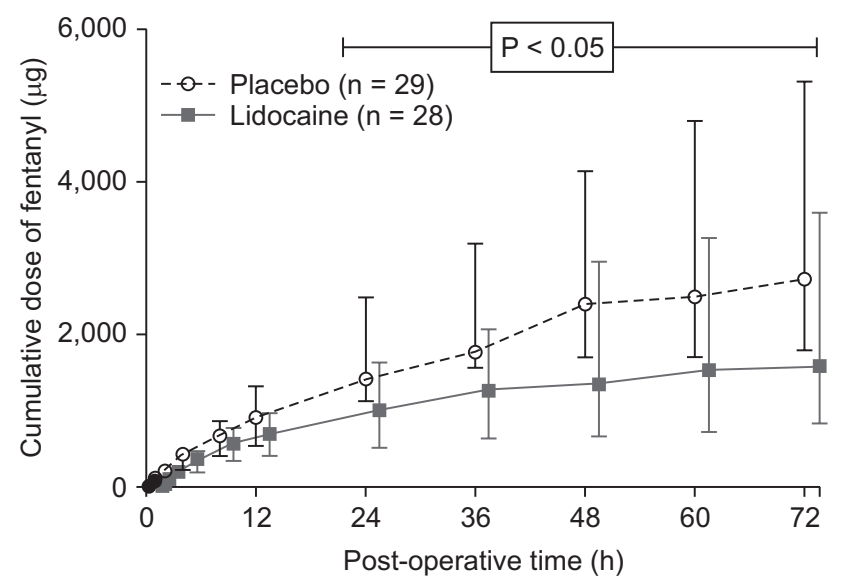

Fig. 3. Cumulative postoperative opioid use ( $\mu \mathrm{g}$ fentanyl). Data are presented as median (IQR). All measured time points from 12 to 72 postoperative hours showed a statistically significant reduction in opioid use $(\mathrm{P}<0.05)$.

Table 2. Time Outcomes between Randomized Arms

\begin{tabular}{lccc}
\multicolumn{1}{c}{ Variables } & Placebo $(\mathrm{n}=29)$ & Lidocaine (n=28) & Difference (95\% CI) \\
\hline Time to first bowel movement (h) & $82.5(40.4)$ & $80.1(42.2)$ & $-2.4(-24.3$ to 19.6$)$ \\
Time to first flatus (h) & $70.0(31.2)$ & $64.7(38.5)$ & $-5.3(-23.9$ to 13.3$)$ \\
Length of hospital stay (days) & $11(9-13)$ & $9(8-14)$ & 0.530 \\
\hline
\end{tabular}

Data are presented as mean (SD) for bowel recovery, and median (IQR) for length of hospital stay. 


\section{Plasma lidocaine levels}

The plasma concentrations of lidocaine taken one hour after initiation were $<0.4 \mu \mathrm{g} / \mathrm{ml}$ in all 14 placebo-controlled patients. In contrast, the concentration of lidocaine was detectable in all the 15 patients receiving active treatment and averaged $1.6(0.6)$ $\mu \mathrm{g} / \mathrm{ml}$. The lidocaine concentration did not exceed the toxicity threshold of $5 \mu \mathrm{g} / \mathrm{ml}$ in any of these patients.

\section{Adverse effects}

Mortality was zero in both groups. The frequency of adverse effects was $46.4 \%$ (13 of 28) and 34.5\% (10 of 29) for the lidocaine and placebo groups respectively, with no significant difference between the groups. None of these identified adverse effects were deemed related to the lidocaine infusion. The most frequent adverse event was nausea and/or vomiting which occurred in $6(21.4 \%)$ and $5(17.2 \%)$ patients receiving lidocaine and placebo, respectively. One patient in the lidocaine group developed sepsis deemed not related to lidocaine treatment, causing a prolonged hospital stay of 118 days. Two patients in the control group displayed transient intra-operative ventricular bigeminy.

\section{Discussion}

This is the first study to investigate the effect of a prolonged 48-hours postoperative lidocaine infusion in patients undergoing abdominal surgery in a double-blind placebo-controlled design. Lidocaine infusion did not significantly reduce time to first bowel movement compared to placebo, nor did it reduce time to first flatus, postoperative pain scores, or length of hospital stay. However, it reduced opioid consumption by up to $40 \%$ from 24-72 hours postoperatively.

The effect of lidocaine on other postoperative measures in our study was less pronounced compared to the recent meta-analysis by Sun et al. [4]. This analysis showed a significant reduction in time to first flatus and bowel movement by 11.11 hours and 15.11 hours respectively; reduced hospital length of stay by 0.71 days; and reduced rest pain scores (visual analog scale, VAS) at 6 hours $(-4.53 \mathrm{~mm}), 24$ hours $(-4.87 \mathrm{~mm})$, but not at 72 hours. However, we note that all 15 trials displayed significant heterogeneity of infusion time, dose and duration, surgery types, and outcome measures, which are summarized in Table 3. Infusions were started pre-operatively in eleven trials and postoperatively in four trials; continuing either until the end of surgery or for 1,4 , or 24 hours postoperatively. Most studies initiated lidocaine with i.v. boluses of $1.5-2 \mathrm{mg} / \mathrm{kg}$ or $100 \mathrm{mg}$, followed by an infusion of $1-3 \mathrm{mg} / \mathrm{kg} / \mathrm{h}$ or $2-3 \mathrm{mg} / \mathrm{min}$ [4]. Only 3 of 6 studies showed significant improvement in bowel flatus recovery, 3 of 4 in bowel movement recovery [8], 1 of 4 faster hospital discharge, 5 of 12 reduced pain scores, and 4 of 11 reduced opioid use outcomes (Table 3). A Cochrane review [9] of 45 trials investigating perioperative i.v. lidocaine efficacy, published after completion of this study, summarizes the limitations of current trials: low-moderate level evidence for pain scores in abdominal surgery; limited evidence on the return of bowel function, hospital discharge, and opioid use; and a scarcity of studies assessing the optimal dose and timing of infusions. This review showed significant reductions in time to first bowel movement (Mean Difference [MD] -6.12 hours, 95\% CI -7.36 to -4.89 ), and pain scores in open abdominal surgery occurred at 24 hours, with an MD of -0.72 ( $95 \%$ CI -0.96 to -0.47$)$ VAS compared to placebo.

Given the heterogeneity of surgery type, we selected for comparison three previous studies $[6,7,10]$ of a similar cohort in open colorectal surgery (Table 3). Two of these studies shared with ours a comprehensive evaluation of bowel function, duration of hospital stay, opioid use, and pain scores [6,7]. Herroeder et al. [6] reported in a similar size study, faster recovery of bowel function and hospital discharge, but no difference in pain scores or opioid consumption. A smaller study of 22 patients by Harvey et al. [7] showed a faster recovery of bowel movement and earlier discharge in the lidocaine group, but no significant differences in flatus recovery, opioid consumption, or VAS pain scores at 6,18 , or 24 hours. In a study of 40 patients, Kuo et al. [10] demonstrated a faster return of flatus, a reduction in early VAS pain scores, but not earlier hospital discharge [10]. Overall, these studies show beneficial effects of i.v. lidocaine on bowel recovery, but not opioid consumption. Ours is the only study among these four reporting reduced opioid consumption in patients undergoing open colorectal surgery. We included a relatively large sample size calculated to give a power of $80 \%$ at a a level of 0.05 to detect a clinically significant shortening of bowel recovery by 12 hours.

There are several possible explanations for observed differences. The Cochrane review [9] and meta-analysis [4] suggest that the lack of uniformity of i.v. lidocaine regimens, surgical types, and outcome measures in existing studies contribute to the heterogeneity of trial results and limited available evidence. While our study showed a non-significant trend towards shorter time to bowel recovery, the variances were larger compared to the other studies $[6,7,10]$, suggesting that our study may have been underpowered to detect a difference. Our trial was conducted between two institutions in patients of three surgeons, whose procedures and approaches were varied. We did not record variance in surgical practice, such as stoma site location. This heterogeneity may explain the large variance in bowel recovery times. Given the lack of information on surgical practice in other studies, it is difficult to assess how this may confound 


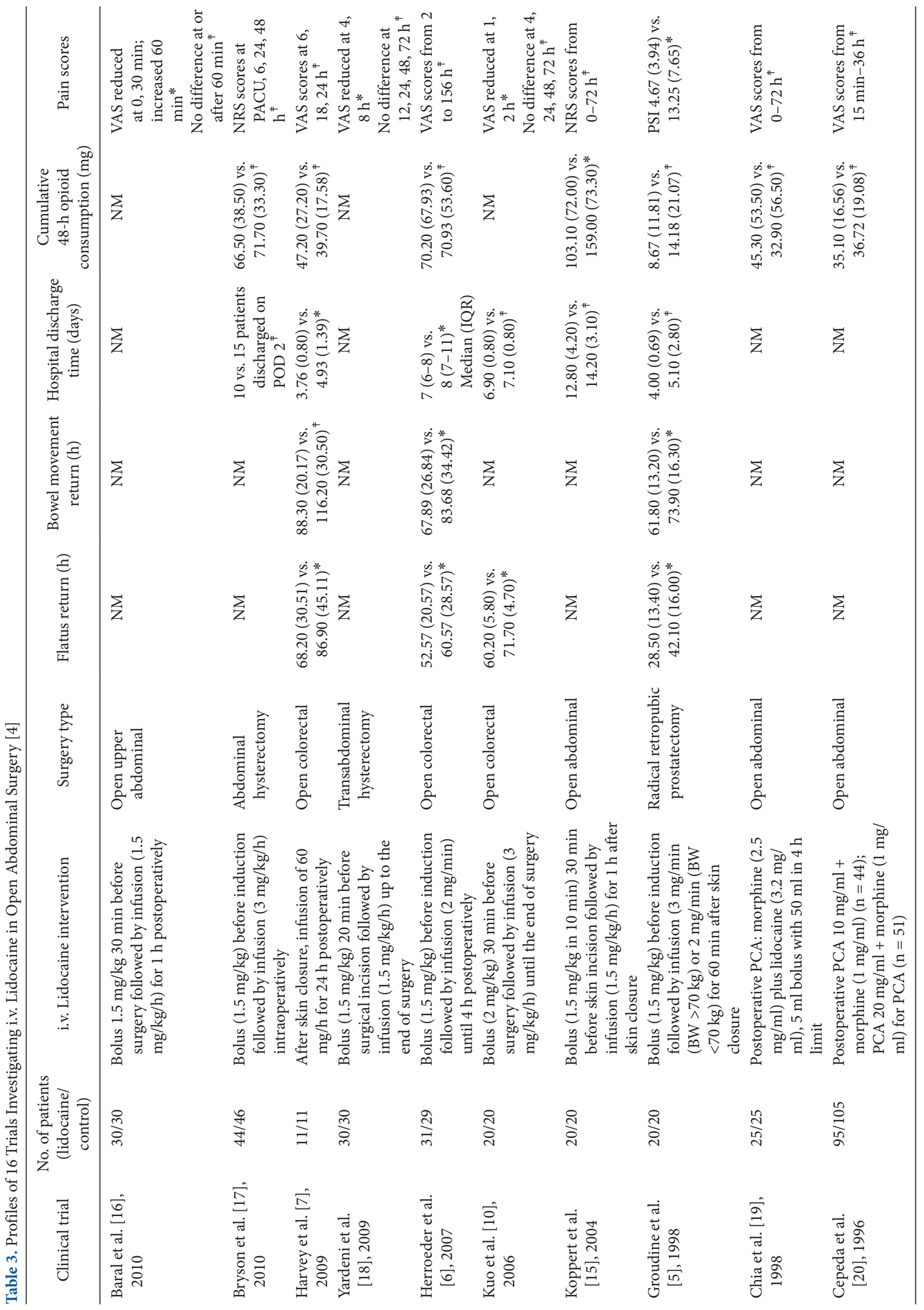




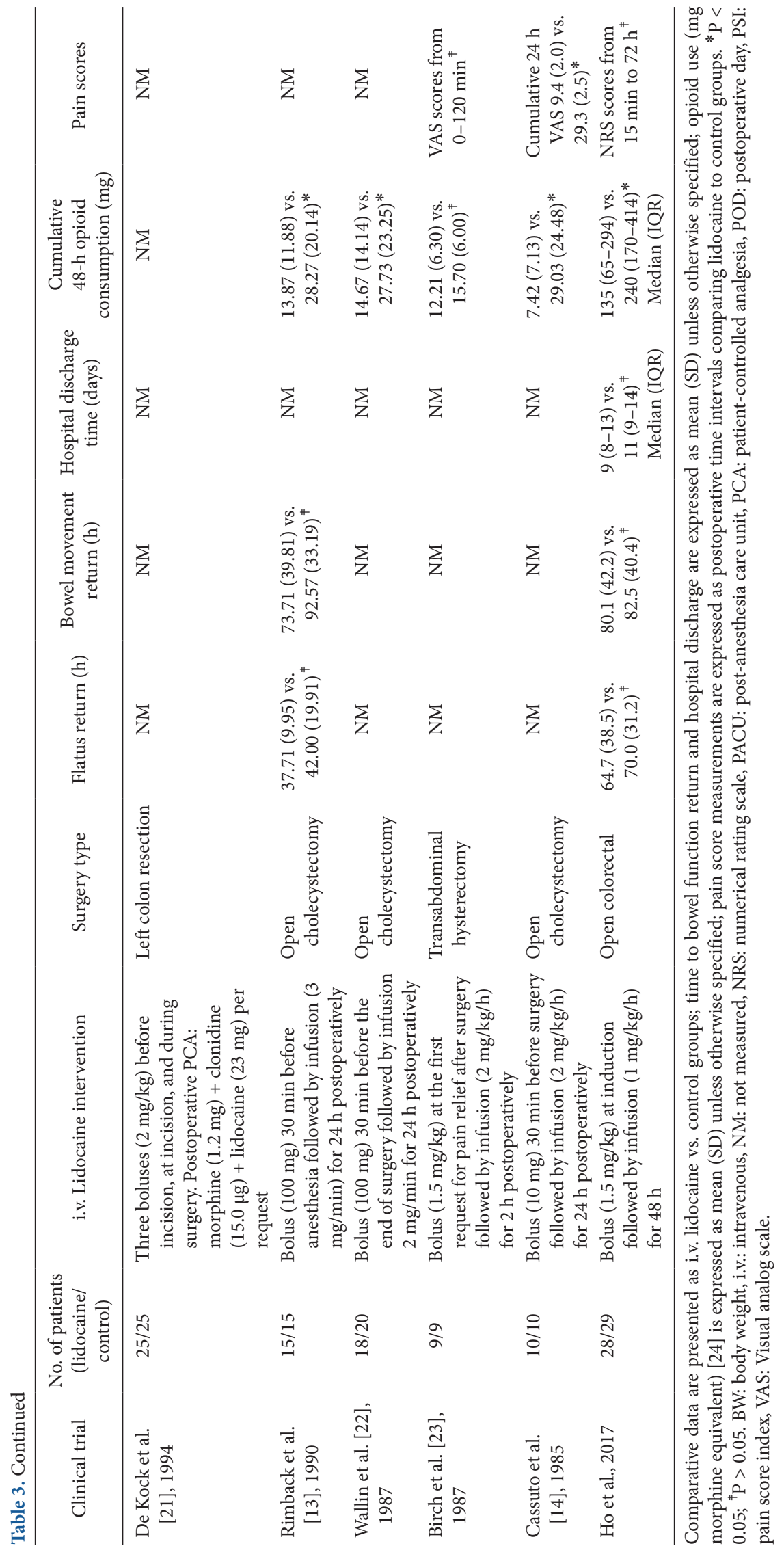


the effects of i.v. lidocaine.

The mechanisms by which lidocaine is proposed to enhance bowel recovery are not well understood but include an opioid-sparing effect, selective suppression of sympathetic inhibitory spinal reflexes mediating ileus [1], selective inhibition of pain transmission in the spinal cord [11], reduction of circulating inflammatory mediators $[10,12]$, and inhibition of the tetrodotoxin-resistant sodium currents that occur with mechanical stretch of bowel. In our study, the opioid-sparing effect of lidocaine was not associated with improvement in bowel recovery time. None of the four previous studies including our own, measuring bowel recovery and opioid use, showed simultaneous reductions in both (Table 3) [5-7,13]. Our negative findings on functional recovery suggest that treatment of postoperative ileus in bowel surgery is not primarily dependent on opioid-sparing techniques. For example, Enhanced Recovery After Surgery protocols harness a diverse range of techniques to enhance bowel recovery, which include avoidance of fasting and bowel preparation, early nutrition, thoracic epidural placement, and early nasogastric tube removal.

This study had several limitations. This was a two-centerstudy involving three surgeons, each with varying peri-operative practice, which may explain the large variance bowel recovery and hospital discharge times. However, the varied surgical environments more closely reflect real clinical practice, compared to other single-centered, single-surgeon studies. Secondly, our i.v. lidocaine infusion dose $(1 \mathrm{mg} / \mathrm{kg} / \mathrm{h})$ was low compared to that of other studies ( $60 \mathrm{mg} / \mathrm{h}$ to $3 \mathrm{mg} / \mathrm{kg} / \mathrm{h}$ ), and limited in order to gain approval by our Human Research Ethics Committee. However, plasma lidocaine concentrations in our study (1.6 $\pm 0.6 \mu \mathrm{g} / \mathrm{ml}$ ) were within the range of four previous studies in which mean values were between 1.3 and $4.2 \mu \mathrm{g} / \mathrm{ml}[5,6,14,15]$. Tanelian and MacIver [11] established that the pain-relieving concentration range required to reduce tonic injury discharge in both $\mathrm{A}-\mathrm{d}$ and C-fibres is $1-15 \mu \mathrm{g} / \mathrm{ml}$. However, in a systematic review, McCarthy et al. [8] note that adequate plasma concentrations did not always correlate with analgesic benefit.

While a recent meta-analysis [4] promotes i.v. lidocaine as a safe and effective peri-operative analgesic, the level of evidence is insufficient to recommend this as superior to conventional peri-operative analgesia for open abdominal surgery. The Cochrane review concludes that further studies with larger numbers are warranted to examine the effect of i.v. lidocaine on bowel recovery in heterogeneous populations; to compare the various infusion protocols with the aim of dose and plasma concentration level optimization; and to compare the efficacy of i.v. lidocaine with other multimodal options, such as epidurals [9].

Postoperative ileus remains a significant morbidity in patients undergoing open colorectal surgery. The pronounced late postoperative opioid-sparing effects shown by this study support the use of longer i.v. lidocaine infusions as an opioid-sparing peri-operative analgesic. Thus, i.v. lidocaine may be more useful for patients in whom high opioid use is likely, or represents particular peri-operative health risks. However, this does not support its routine use to speed bowel recovery after open bowel surgery.

\section{Acknowledgments}

The authors wish to acknowledge the support for this study given by staff from the Department of Anesthesia, St Vincent's Hospital, Sydney; the Departments of Research, Anaesthesia and Surgery at St Vincent's Private Hospital, Sydney; Drs. Rohan Gett, Alan Meagher, and Gareth Owen from the Department of Surgery, St Vincent's Hospital, Sydney; Bridget Prior and Kelly Stallard for assistance with data collection; Anita Matijasko, Jed Duff, and Edel Murray for organization of study interventions; and Dr. Erez Ben-Menachem for manuscript advice.

This study was supported by internal funds from the Department of Anaesthesia, St Vincent's Hospital, Sydney, which contributed to study drug and infusion equipment costs. The authors did not receive any specific funding for this study.

\section{ORCID}

Matthew Liang Jinn Ho, https://orcid.org/0000-0002-3152-6473 Stephen John Kerr, https://orcid.org/0000-0002-1919-4525

Jennifer Stevens, https://orcid.org/0000-0001-9837-0031

\section{References}

1. Nadrowski L. Paralytic ileus: recent advances in pathophysiology and treatment. Curr Surg 1983; 40: 260-73.

2. White PF. The role of non-opioid analgesic techniques in the management of pain after ambulatory surgery. Anesth Analg 2002; $94: 577-85$.

3. Barletta JF, Asgeirsson, Senagore AJ. Influence of intravenous opioid dose on postoperative ileus. Ann Pharmacother 2011; 45: 916-23.

4. Sun Y, Li T, Wang N, Yun Y, Gan TJ. Perioperative systemic lidocaine for postoperative analgesia and recovery after abdominal surgery: a meta-analysis of randomized controlled trials. Dis Colon Rectum 2012; 55: 1183-94.

5. Groudine SB, Fisher HA, Kaufman RP Jr, Patel MK, Wilkins LJ, Mehta SA, et al. Intravenous lidocaine speeds the return of bowel function, decreases postoperative pain, and shortens hospital stay in patients undergoing radical retropubic prostatectomy. Anesth Analg 1998; 86: 
235-9.

6. Herroeder S, Pecher S, Schönherr ME, Kaulitz G, Hahnenkamp K, Friess H, et al. Systemic lidocaine shortens length of hospital stay after colorectal surgery: a double-blinded, randomized, placebo-controlled trial. Ann Surg 2007; 246: 192-200.

7. Harvey KP, Adair JD, Isho M, Robinson R. Can intravenous lidocaine decrease postsurgical ileus and shorten hospital stay in elective bowel surgery? A pilot study and literature review. Am J Surg 2009; 198: 231-6.

8. McCarthy GC, Megalla SA, Habib AS. Impact of intravenous lidocaine infusion on postoperative analgesia and recovery from surgery: a systematic review of randomized controlled trials. Drugs 2010; 70: 1149-63.

9. Kranke P, Jokinen J, Pace NL, Schnabel A, Hollmann MW, Hahnenkamp K, et al. Continuous intravenous perioperative lidocaine infusion for postoperative pain and recovery. Cochrane Database Syst Rev 2015; (7): CD009642.

10. Kuo CP, Jao SW, Chen KM, Wong CS, Yeh CC, Sheen MJ, et al. Comparison of the effects of thoracic epidural analgesia and i.v. infusion with lidocaine on cytokine response, postoperative pain and bowel function in patients undergoing colonic surgery. Br J Anaesth 2006; 97: 640-6.

11. Tanelian DL, MacIver MB. Analgesic concentrations of lidocaine suppress tonic A-delta and C fiber discharges produced by acute injury. Anesthesiology 1991; 74: 934-6.

12. Kawamata M, Takahashi T, Kozuka Y, Nawa Y, Nishikawa K, Narimatsu E, et al. Experimental incision-induced pain in human skin: effects of systemic lidocaine on flare formation and hyperalgesia. Pain 2002; 100: 77-89.

13. Rimbäck G, Cassuto J, Tollesson PO. Treatment of postoperative paralytic ileus by intravenous lidocaine infusion. Anesth Analg 1990; 70: 414-9.

14. Cassuto J, Wallin G, Högström S, Faxén A, Rimbäck G. Inhibition of postoperative pain by continuous low-dose intravenous infusion of lidocaine. Anesth Analg 1985; 64: 971-4.

15. Koppert W, Weigand M, Neumann F, Sittl R, Schuettler J, Schmelz M, et al. Perioperative intravenous lidocaine has preventive effects on postoperative pain and morphine consumption after major abdominal surgery. Anesth Analg 2004; 98: 1050-5.

16. Baral BK, Bhattarai BK, Rahman TR, Singh SN, Regmi R. Perioperative intravenous lidocaine infusion on postoperative pain relief in patients undergoing upper abdominal surgery. Nepal Med Coll J 2010; 12: 215-20.

17. Bryson GL, Charapov I, Krolczyk G, Taljaard M, Reid D. Intravenous lidocaine does not reduce length of hospital stay following abdominal hysterectomy. Can J Anaesth 2010; 57: 759-66.

18. Yardeni IZ, Beilin B, Mayburd E, Levinson Y, Bessler H. The effect of perioperative intravenous lidocaine on postoperative pain and immune function. Anesth Analg 2009; 109: 1464-9.

19. Chia YY, Tan PH, Wang KY, Liu K. Lignocaine plus morphine in bolus patient-controlled intravenous analgesia lacks post-operative morphine-sparing effect. Eur J Anaesthesiol 1998; 15: 664-8.

20. Cepeda MS, Delgado M, Ponce M, Cruz CA, Carr DB. Equivalent outcomes during postoperative patient-controlled intravenous analgesia with lidocaine plus morphine versus morphine alone. Anesth Analg 1996; 83: 102-6.

21. De Kock M, Lavandhomme P, Scholtes JL. Intraoperative and postoperative analgesia using intravenous opioid, clonidine and lignocaine. Anaesth Intensive Care 1994; 22: 15-21.

22. Wallin G, Cassuto J, Högström S, Lindén I, Faxén A, Rimbäck G, et al. Effects of lidocaine infusion on the sympathetic response to abdominal surgery. Anesth Analg 1987; 66: 1008-13.

23. Birch K, Jørgensen J, Chraemmer-Jørgensen B, Kehlet H. Effect of i.v. lignocaine on pain and the endocrine metabolic responses after surgery. Br J Anaesth 1987; 59: 721-4.

24. Lin YM, Fu Y, Winston J, Radhakrishnan R, Sarna SK, Huang LM, et al. Pathogenesis of abdominal pain in bowel obstruction: role of mechanical stress-induced upregulation of nerve growth factor in gut smooth muscle cells. Pain 2017; 158: 583-92. 